\title{
Risk Analysis Techniques in Construction Engineering Projects
}

\author{
Daniel Baloi \\ Department of Civil Engineering, Eduardo Mondlane University, Av. de Moçambique, km 1,5, Maputo, Mozambique \\ Email: Baloi@zebra.uem.mz \\ Received 18 April 2011 \\ Accepted 8 June 2012
}

\begin{abstract}
There is a great deal of risk analysis techniques and tools available for the management of risks. In principle, each risk analysis technique has its strengths and weaknesses. Techniques such as Probability Theory, Certainty Factors, Dempster-Shaffer Theory of Evidence and Fuzzy Logic are discussed with regard to their application to risk analysis in construction engineering projects. Suggestions on the most appropriate tools associated with the techniques are also presented. The strengths and weaknesses of each technique are highlighted and discussed. It has been concluded that the nature of risks under consideration is determinant in the selection of modeling and analysis techniques.
\end{abstract}

Keywords: Risk analysis, construction, projects, techniques, uncertainty

\section{Introduction}

Risk management constitutes one of the key elements towards effective project implementation and success. The risk management process can be viewed in different ways. From the project management perspective it is common to consider planning, identification, classification, analysis, response, monitoring and control as the main steps. All these steps are interconnected and are part of a system, which means each should be properly addressed so as to enable an effective operation of the whole. Risk analysis aims to estimate or assess the likely outcomes or impacts of risks under consideration, in case they materialize. Deciding which courses of action to pursue, risk response strategies, is largely based on the results of risk analysis. There is a great deal of risk analysis techniques and tools available. In principle, each risk analysis technique has its strengths and weaknesses and, as such, the option for one specific technique depends on several factors of which the effectiveness to capture the inherent uncertainty is of paramount importance. Very often, though, the selection of a specific modeling and analysis technique is more governed by factors such as familiarity, simplicity and availability, rather than the nature of prevailing risks combined with the power to describe uncertainty. This procedure is likely to hinder the validity of the outcomes seriously and, consequently, the whole risk decision-making process.

There are instances where qualitative techniques are more effective than quantitative, although the latter may appear to be the most robust and meaningful for many practitioners. Again, the right analysis technique is the one capable of adequately capturing and handling uncertainty. The purpose of this paper is to review and discuss risk analysis techniques that would be most suitable to construction management.
Some of the risk analysis techniques available include preliminary risk analysis, faulty trees, event trees, sensitivity analysis, probability analysis, certainty factors, DempsterShaffer Theory of Evidence and Fuzzy Logic. Four of these methods, probability analysis, certainty factors, DempsterShaffer Theory of Evidence and Fuzzy Logic are discussed with regard to their application to risk analysis in engineering projects. The main reason for considering these four techniques is their potential to effectively handle uncertainty inherent in construction projects. The study analyzes how suitable the techniques are for the risk categories under consideration, namely organization-specific, global and Acts of God. Among these, Acts of God are obviously handled in a very different manner as 'force majeure" under contractual terms. The strengths and weaknesses of the techniques are highlighted and discussed. The remaining techniques are not considered since they rarely apply to project risk management. They are more appropriate for problems commonly found in fields such as safety/hazard, biological, pharmaceutical and health.

This work is part of a larger study conducted to develop a framework for managing risk factors affecting construction projects costs. The actual detailed modeling and analysis, including computations, are not part of the present paper due to scope delimitation and space limitations. They will be discussed in the next stage. Although the study concentrates on construction engineering project risk, it is believed that the risk analysis techniques under consideration can be employed in various other fields as they are cross-cutting.

\section{Risks in Construction Projects}

Construction projects as other types of projects pose serious management challenges. Geographical dispersion, significant number of players, technical variability, technical complexity 
and large number of inputs are some of the variables that make construction projects challenging. As a result of these interconnections the number and type of risks tend to be significant. Risk classification is not a consensual exercise even among the construction community but the most important issue is the recognition of risks and the need to tackle them so as to increase the chance of project success. Many different classifications of risk have been developed. However, most of these have considered the source as the most important criterion. Following this criterion, one way of classification for construction is as follows: technical; construction; legal; natural; logistic; social; economic; financial; commercial; and political, Thompson and Perry ${ }^{1}$. It is likely that apart from construction, these categories of risks apply to a wide variety of other projects such as mineral, manufacturing and transport.

A classification taking into account the location of the impact of risks in the elements of the project has been suggested by Flanagan and Norman ${ }^{2}$. It is also usual to categorize risks on a wider perspective as dynamic/static, corporate/individual, internal/external, positive/negative, acceptable/unacceptable, short term/long term and insurable/non-insurable.

The adoption of source as a means to risk classification gives rise to a myriad of diverse risks that need to be properly managed to enable effective responses delineation. Political risks can hardly be handled, in terms of modeling, measurement of likelihood and impact, in the same way as the technical ones, for example. From this reasoning, it follows that it is fundamental to identify the most effective techniques to perform the operation.

\section{Nature of Construction Projects Risks}

As it has been discussed, construction project risks were classified in accordance with source as technical, legal, natural, logistic, social, economic, financial, commercial and political. In order to facilitate the analysis and reduce the number of classes, these risks can be grouped at a higher level in the breakdown structure using the concept of environment layers. Before that is done it is important to present some important concepts.

All organizations exist within an environment and not in a vacuum. The structure of the environment surrounding a project or organization can be subdivided into three distinct layers: outer layer or general environment; operational environment; and inner layer or internal environment. Both the general and operational environments can be considered external environments. The general environment comprises domains that are broad in scope and have little immediate and direct impact on the organization's activities. The general environment comprises five basic elements or domains, namely economic environment, political environment, social environment, technological environment and physical or natural environment. These domains interact with organizations or projects constantly and determine what they "might do". In general, some environment domains are less predictable and difficult to understand than others.

The operating environment is the external environment comprising factors that have more specific and immediate impact on the organization or project. These factors include suppliers, clients, sub-contractors, consultants and competitors. Finally, the internal environment is the inside environment that has direct, close, and immediate impact on the organization. The internal environment is basically concerned with organization's resources, which include financial, physical, human, and technological resources, as well as managerial values and ethics.

Physical resources in construction include labor, equipment and materials. System and technological resources are technical capabilities and models used in the operations of the organization, such as quality control processes, reward policies, patents, brands and technologies. In conclusion, the internal environment of an organization identifies its strengths and weaknesses, i.e., what the organization "can do".

Based on both opportunities/threats/constraints from the external environment and strengths/weaknesses/constraints from the internal environment the organization determines "what should be done". Opportunities and threats are current or future conditions in the environment, whereas strengths and weaknesses are positive and negative internal conditions of the organization. In this context, opportunities/threats/constraints from the external environment and strengths/weaknesses/constraints from the internal environment are strongly related to risks.

Following the concept of environmental layers approach, project risks are assumed to be associated with the internal, operational and general environments. Projects risks are, therefore, classified according to their primary source or cause and the environmental layers as:

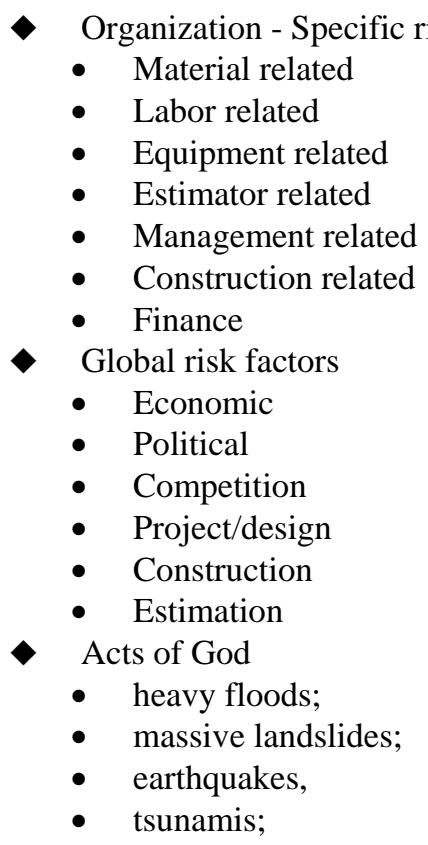


- hurricanes and

- others.

This classification is important for risk decision making process, since managers are expected to carefully examine both internal and external environments of their organizations or projects in order to evaluate the factors that pose serious challenges to success. The consideration of the relevant primary sources of risks and the subsequent detailed examination of the risks linked to each primary source is crucial in any project.

\section{Uncertainty Categories}

Understanding uncertainty associated with risk categories is crucial for their effective management. Uncertainty in the context of the present work refers to lack of sureness about a phenomenon, process, system or event, and can vary from partial sureness to a complete lack of conviction. There are various types of uncertainty inherent in decision-making problems. More often than not decision-making is a challenging endeavor because data and information are scarce, incomplete, inaccurate or unavailable.

Research on uncertainty types has been conducted over time, but the advances in computing technologies were a significant boost. In recent years, a great deal of knowledge on uncertainty has been produced. Helton ${ }^{3}$, suggested that there are two main types uncertainty, namely random and epistemic. Random uncertainty results from the random behavior of a system. This type of uncertainty is also known as aleatory, stochastic, irreducible, objective or variability. Random uncertainty can be described as unknowns that differ each time an experiment is made. This kind of uncertainty cannot be minimized by more precise and accurate measurements. However, the likelihood of specific outcomes occurring can be obtained. According to $\mathrm{Klir}^{4}$, randomness refers to a situation where the outcomes of an event are rigorously a matter of chance, that is, it is impossible to predict the outcomes. For example, casting a die has several possible outcomes each with known probability (perfect die) or with unknown probability (defective die).

Epistemic uncertainty, on the other hand, originates from lack of knowledge about a system, object or process. It is also called reducible, subjective, state of knowledge or ignorance. Epistemic or systematic uncertainty derives, to a greater extent, from lack of knowledge about a phenomenon, system or process. Very often efforts are directed to turning epistemic into random uncertainty, at least, which has proved a daunting challenge.

Van Gelder ${ }^{5}$ grouped uncertainty into natural variability and knowledge uncertainty, respectively. This classification is similar to Helton's, where variability and knowledge are equivalent to random and epistemic, respectively. Subcategories of variability uncertainty include temporal, spatial and individual heterogeneity, whereas knowledge encompasses model, parameter and decision-related uncertainty.

Model-related uncertainty arises from oversimplification or from failure to capture important characteristics of the process/system. Furthermore, failure to understand uncertainty can lead to significant errors due misrepresentation. Surrogate variables, excluded variables, abnormal situations, approximation and incorrect form constitute the main sources of model uncertainty. Natural variability uncertainty cannot be reduced as it stems from the variability of natural forces. That is the reason why it is also called irreducible.

Another perspective on uncertainty defines vagueness and randomness, Zadeh ${ }^{6}$. Vagueness arises when the meaning of a statement or word is poorly-defined, that is, it lacks precision or sharpness. For example, risk factor "strong competition" does not have an exact meaning, because the qualifier 'strong" may assume several degrees of intensity. Strong competition may involve a wide spectrum of human perceptions. Therefore, there is no rigorous definition of what "strong competition""" is.

A particular type of vagueness is fuzziness. Fuzziness is a kind of imprecision where the transition from a membership state to a non-membership of an element to a set is gradual. Fuzziness is a general characteristic in many areas such as management, engineering, manufacturing, and medicine. It is, nevertheless, most frequent in situations where human judgment is an essential feature such as reasoning, learning and decision-making process as suggested by Zimmermann ${ }^{7}$. From the review, it can be concluded that there are several types of uncertainty associated with natural, managerial, social, economic, technological and political phenomena. The three groups of construction engineering risk factors differ in nature and the underlying uncertainty. Assessing the nature of uncertainty is, therefore, crucial for deciding how modeling should be conducted in order to cater for uncertainty attached to risk factors.

\section{Appropriateness of Techniques for Risk Modeling and Analysis}

The different groups of risks that permeate construction projects have been presented and briefly described. Risk managers have been confronted with the choice of right risk analysis technique. Is it effective to employ the same technique to model organization-specific, global and Acts of Gods risk-related factors? The response is not straightforward, but it appears that due to the difference in these risks nature, the underlying uncertainty is also likely to differ. For example, uncertainty attached to Acts of God and organization-specific risks are hardly similar. While phenomena such as earthquakes and 200-year period return river discharge are highly unpredictable (stem from natural variability), the impacts of financial performance or human 
resources competence on a project being implemented by an organization can be reasonably assessed.

The following section discusses the effectiveness of four risk analysis techniques to tackle uncertainty inherent in organization-specific, global and Acts of God related risks. Furthermore, suggestions on the most appropriate tools that can be used to materialize the above mentioned analysis techniques are briefly advanced. The analysis is expected to shed some light on the diversity of modeling and analysis perspectives as well as their effectiveness.

\section{Probabilistic Analysis Technique}

It is commonplace to model and analyze risks through available specific techniques/tools. Most of the tools available in the market are based on probability theory. As such, probabilistic modeling and analysis has been the prominent way to handle risks regardless their nature. Those who argue in favor of probability theory as the foremost technique state that random methods are the only effective methods for dealing with uncertainty. On the other hand, Uher and Toakley $^{8}$ noted that the uncertainty inherent in real risk situations was epistemic rather than aleatoric (matter of chance). This view corroborates the general perception that risk management in construction engineering is eminently cognitive. Indeed, the process is mainly based upon experience, assumptions and human judgment.

It appears that reliance on the probability theory as the only effective and reliable methodology to deal with uncertainty has historical roots. Indeed, probability theory has wellestablished and sound scientific foundations and has been widely used for centuries in all spheres of science.

Probability is a branch of mathematics concerned with random phenomena. As such, it deals with stochastic processes and events through frequentist, outcomes of repeated experiments, and subjective views. Probability theory has been widely used to model precisely described, repetitive experiments with observable but uncertain outcomes.

\subsection{Probability Basics}

The basic assumption in the classical theory of probability is that all types of uncertainty are "'frequentist" measures of randomness or subjective measures of confidence. This reasoning is clear in the definition of probability, as follows: "if a random experiment has $\mathbf{N}$ possible outcomes which are all equally likely and mutually exclusive and $\mathbf{n}$ of these possibilities have outcome $\mathbf{A}$, then the probability of outcome A is $n / N^{\prime \prime}$. The basics of probability theory is, thus, the elicitation of probabilities, which may either be objective or subjective, in order to predict the likelihood of uncertain events. Objective probabilities are those directly derived through experiments or statistical data. Objective probabilities apply to repeatable events only. Subjective probabilities, on the other hand, represent degrees of belief of the decisionmakers. Individuals with diverse backgrounds are entitled to assess the likelihood of the same events differently.

\subsection{Organization-specific Risks}

Organization-specific risks are related to the internal environment of organizations and these are supposed to manage them. Organizations' level of knowledge about the operations is significantly higher than other type of risk. Indeed, issues such as planning, finance, human resources, equipment and materials logistics are dealt with on a daily basis. Organizations can conduct operations studies in order to build robust databases and learn systematically. Very often databases are used as a means for routine programmed decision-making. The large quantity of information obtained in this way can be retrieved in order to make probabilistic estimates. For example, important aspects such as labor productivity and cost can be estimated in probabilistic terms in the planning process through probability distributions. From this point of view, it appears that probabilistic modeling and analysis is suitable for this kind of risks. As a great deal of knowledge is available, predictions about the likelihood and impacts of risks can be made with some degree of certainty. The prominent uncertainty associated with organization-related risks is assumed to be eminently random. In term of tools, simulation and analytical models can be utilized for risk modeling analysis.

One of the most effective tools for this purpose would be Decision Support Systems - DSS, which are computer information systems that provide information in a given domain of application by means of analytical decision models and databases, in order to support a decision-making in complex and ill-structured problems.

\subsection{Global Risk Factors}

Global risk factors are beyond organizations' control. They relate to a more complex, erratic and dynamic environment. As such, the possibility for collecting data and information in order to draw useful lessons becomes limited. Even taking into account the cyclic nature of some factors such as economy ups and downs, it is very difficult to make reasonable estimates about them. Although there have some attempts to estimate the degree of risk associated with global risks the amount of available data make the exercise difficult. An example of probabilistic modeling applied to global risk factors is the production of political risk indices covering a large number of countries around the world as explained by Bremmer $^{9}$. The exercise has been conducted and the results marketed systematically over the years. The indices measure the stability perception over specific countries and aim to help investors and other interested parties in their business 
decision making. Political variables are complex and dynamic and, as consequence, very difficult to grasp. In general, political phenomena are rarely repetitive, which creates serious challenges for effective modeling. The same applies to the economic and social variables. Furthermore, political, economic and social events are rarely mutually exclusive, exhaustive and conditionally independent. First, there have been strong interrelationships among these variables. Economy, for example, is strongly associated with social and political events. In the light of these characteristics it can be concluded that probabilistic modeling of global risk factors poses serious challenges in terms of robustness and validity. The uncertainty inherent in this group of risk factors is much more epistemic rather than random.

\subsection{Acts of God}

Acts of God are generally classified as "force majeure" under the contractual terms, because no party is supposed to be able to manage them and, consequently, bear the associated costs. They represent extreme events stemming from nature and, as such, tremendously difficult to predict. It would be important for a contractor, for example, to know the likelihood of an earthquake striking and disrupting construction works. Unfortunately, such estimates are not available. That is the reason why the best response strategy to this type of risk has been transfer to a third party.

Attempts have been made to predict the probability of occurrence of these events, but very little progress has been achieved so far. Concerns over the problem have led analysts to develop some probabilistic models but, it should recognized, these can hardly be robust due to prevailing nature of uncertainty. Therefore, such estimates may be precise but not accurate. In addition, since most nature-related risk factors are poorly defined and fuzzy in nature they cannot be evaluated with such high precision inherent in numerical expressions. These models have used subjective probabilities instead of objective ones, as the latter are difficult to obtain due to lack of data. The fact that subjective probabilities cannot be distinguished from objective ones once in the model, is likely to hinder the quality of the analysis. There is guarantee that subjective probabilities capture uncertainty effectively. As a result, probabilistic modeling and analysis of nature-related risks is debatable.

\section{Certainty Theory Analysis Technique}

\subsection{Global Risk Factors and Acts of God}

As previously shown, while organization-related risk factors can be well modeled through probabilistic means, global risks and Acts of God are far more difficult to handle in the same way due to the inherent kind of uncertainty and scarcity of data and information.
Certainty theory is mainly a theory for handling uncertainty in knowledge based systems - KBSs. It was developed in attempt to overcome some of the weaknesses of the so called "idiot Bayes" approaches for inexact reasoning, according to Duda. ${ }^{10}$ Certainty theory relies on defining judgmental measures of belief rather than adhering to strict probability estimates. Therefore, certainty factors (CF) are not probabilities but informal measures of confidence for a piece of evidence. They represent the degree to which people believe that the given evidence is true. In other words, they express how accurate, reliable, truthful people judge statements or evidences.

Certainty theory fundamentals are the concepts of "certainty measures" which are associated with "factual statements". The certainty measures or factors CFs consist of numbers ranging from -1 to +1 and factual statements, (rules). A negative value of the certainty factor indicates that one believes that a fact is not true and a positive value indicates the one believes that a fact is true with complete knowledge.

$\mathrm{CF}=1$, there is complete certainty that a proposition is true

$\mathrm{CF}=-1$, there is complete certainty that a proposition is false

$\mathrm{CF}=0$, there is no information at all about or no change in belief

$-1<\mathrm{CF}<1$, measure of the degree of belief about the proposition with decreasing and increasing beliefs respectively.

Global risk factors and Acts of God in engineering projects can be modeled using CFs within knowledge-based systems, where the following format is common: If $A$ Then $B$ with certainty factor $\mathrm{CF}=\mathrm{CF}$ (rule), where $A$ is the antecedent and $B$, the consequent. The antecedent comprises facts (evidence) that support the derivation of the consequent (hypothesis). The CF is the net degree of belief in hypothesis, given that the evidence is observed (given).

For example: If the rate of inflation increases then the prices will be high $\mathrm{CF}=1$, where the rate of inflation increases is the evidence and the prices will be high is the consequence. The degree of belief associated with the rule is 1 , which means the analyst is $100 \%$ sure. In a typical knowledge based system there are numerous rules of this kind that cover most of the project situations. For global risk factors alone, hundreds of rules would be required in the KBSs.

The formal representation of evidence for, against the hypothesis and the composite certainty factors, that is degree of belief MB, degree of disbelief MD and CF, is defined in terms of hypothesized mathematical relationships governed 
by prior and posterior probabilities of the propositions that they describe, as stated by Clark ${ }^{11}$.

In practical applications of certainty factors rules, hypothesis and input data have an associated MB and MD. Then, the composite certainty factor CF is calculated by aggregating the values of $\mathrm{MB}$ and $\mathrm{MD}$. If the information contained in the premise is not known with certainty $(\mathrm{CF}<1)$, then the level of belief in the conclusion is reduced. The original function used in the aggregation is as follows:

$$
\mathrm{CF}[\mathrm{h}, \mathrm{e}]=\mathrm{MB}[\mathrm{H}, \mathrm{E}]-\mathrm{MD}[\mathrm{H}, \mathrm{E}] \text {; }
$$

This original formula is very often modified for convenience and it becomes

$$
\begin{gathered}
\mathrm{CF}[\mathrm{H}, \mathrm{E}]=(\mathrm{MB}[\mathrm{H}, \mathrm{E}]-\mathrm{MD}[\mathrm{H}, \mathrm{E}]) \text { : } \\
\{1-\min (\mathrm{MB}[\mathrm{H}, \mathrm{E}], \mathrm{MD}[\mathrm{H}, \mathrm{E}])\} .
\end{gathered}
$$

where $\mathrm{CF}[\mathrm{H}, \mathrm{E}]$ is the certainty factor of the hypothesis $\mathrm{H}$ given the evidence $\mathrm{E}, \mathrm{MB}$ the degree of belief, $\mathrm{MD}$ the degree of disbelief in h given $\mathrm{E}$. Both $\mathrm{MB}$ and $\mathrm{MD}$ values vary from 0 to 1 . CF is also known as the composite certainty factors and varies between -1 and +1 . The value of $M B$ and MD are calculated as explained in detail by Shortliffe and Buchanan:

$$
\begin{gathered}
\mathrm{MB}[\mathrm{H}, \mathrm{E}]=1 \text {, if } \mathrm{P}[\mathrm{H}]=1 \\
\mathrm{MB}[\mathrm{H}, \mathrm{E}]=\{\mathrm{P}[\mathrm{H} \mid \mathrm{E}]-\mathrm{P}[\mathrm{H}]\}: \\
\{1-\mathrm{P}[\mathrm{H}]\}, \text { if } \mathrm{P}[\mathrm{H}] \neq 1 \\
\mathrm{MD}[\mathrm{H}, \mathrm{E}]=1 \text {, if } \mathrm{P}[\mathrm{H}]=0 \\
\mathrm{MD}[\mathrm{H}, \mathrm{E}]=\{\mathrm{P}[\mathrm{H}]-\mathrm{P}[\mathrm{H} \mid \mathrm{E}]\}: \\
\mathrm{P}[\mathrm{H}], \text { if } \mathrm{P}[\mathrm{H}] \neq 1
\end{gathered}
$$

where $\mathrm{P}[\mathrm{H}]$ is prior probability of an hypothesis $\mathrm{H}$; $\mathrm{P}[\mathrm{H}, \mathrm{E}]$ is the posterior probability of the hypothesis given some evidence E.

\subsection{Tools}

Knowledge Based Systems - KBSs appear to be the more appropriate tools for the application of certainty factors due to the way knowledge (rules) is represented within the system. Knowledge based systems are particular applications of the DSSs. Essentially, a knowledge based system is a computerized system that uses knowledge about some domain to arrive at a solution to a problem from that domain.

\subsection{Summary}

There are several weaknesses associated with certainty factor theory. First, the measures of belief seem to be largely subjective. They depend on the individual making the assessment and can vary for same individual based on specific circumstances. Therefore, it is not easy to ascertain how accurate the measures are. Blockely and Baldwin ${ }^{12}$ argued that certainty factor theory is an ad-hoc approach and therefore, lacking a formal foundation. The authors criticized the use of parallel combination function for assuming conditional independence and the negation of each hypothesis evidence and considered the theory, worse than the "idiot
Bayes model" which assumes only one of these propositions. Additionally, it appears that the theory does not suit rulebased models because of its non-modular operational requirements. Furthermore, it has been suggested that the CF formulae have little validity and the consultation in CF logic mode tends to require more work on the user's part than is required by the normal Binary logic mode.

\section{Dempster-Shafer Theory of Evidence Analysis Technique}

Dempster-Shafer ${ }^{13}$ theory of evidence is usually called epistemic probability because it provides an alternative model for the assessment of numerical degrees of belief. In principle, it helps to overcome the handicaps of Certainty Factors and can be applied to model phenomena whose behavior falls under epistemic uncertainty. Indeed, DempsterShafer attempts to distinguish between uncertainty and ignorance. It uses belief functions instead of probabilities. In this sense it can be considered a generalization of the Bayesian theory of subjective probability and that is why it is also called theory of belief functions. These degrees of belief may or may not have the mathematical properties of probabilities. The theory gained momentum in the 80's when researchers made some attempts to adapt probability in expert systems.

The Dempster-Shafer theory is based on two core ideas, namely obtaining degrees of belief for one question from subjective probabilities for a related question, and Dempster's rule for combining such degrees of belief when they are based on independent items of evidence.

To illustrate how it works, a company may be interested to assess the fiscal policy risk in a region or a country where it is executing a project. This is usually a crucial factor as project financial/economic viability can be largely influenced. It is obvious that objective probabilities for this kind of risk are hard to estimate. The company can, therefore, resort to subjective probabilities and then develop belief functions. The question being asked would be " is fiscal stability and conducive to business?'”. If this probability is set at $90 \%$, for example, then, the probability of the contrary (unstable and detrimental to business) would be $10 \%$. In such circumstances, the company can be confident that the system is credible and good. The degree of belief attached to the system's reliability is then 0.90 and the degree of belief that it is unreliable is consequently 0.10 . A second question would be - 'was a specific corporate taxation rate fair/balanced?'. Based on the inherent credibility, the fairness of the taxation justifies a degree of belief of 0.90 . The degree of belief of it being unfair is 0.0 and not 0.10 . The later degree of belief does not necessarily mean sureness about an unlikely unfairness. It simply means that the reliability of the policy gives no reason to believe the contrary. The numbers 0.90 and 0.0 together constitute a belief function. The illustration 
presented explains the above-mentioned fundamentals of Dempster-Shafer Theory, namely the degrees of belief attached to two related questions and the rules for combining them. The degrees of belief associated with the specific taxation fairness are determined on the basis of the reliability of the policy/system which has an initial degree of belief.

Dempster-Shafer theory provides a platform (rules) for combining various degrees of belief. For example, different risks with potential to influence project success can be considered for assessment. Having attached degrees of belief on these variables would enable development and combination of belief functions following appropriate rules in order to ascertain the likelihood and impact of different factors. The main assumption behind the rule is the a priori independence of the questions for which probabilities have been estimated.

\subsection{Tools}

Knowledge Based Systems - KBSs appear to be the more appropriate tools for the application of certainty factors due to the way knowledge (rules) is represented within the system.

\subsection{Summary}

The main distinctions between Bayesian models of numerical degrees and Dempster-Shafer model are the following: belief functions of Dempster-Shafer are set functions rather than point values; rejection of the law of additivity for belief in disjoint propositions; and Dempster-Shafer theory has an operation for the pooling of evidence from various sources. Dempster-Shafer theory is richer in terms of semantics since it allows an expression of partial knowledge. Its main shortcoming is, however, the elicitation and interpretation of belief functions. Furthermore, the computational methods employed in the theory are very complex and thus, of little practical use. This is the reason why it has had very little application.

\section{Fuzzy Set Analysis Technique}

Fuzzy set theory is a branch of modern mathematics that was formulated by Zadeh ${ }^{14}$ to model vagueness intrinsic to human cognitive processes - humanistic systems. Since then, it has been used to tackle poorly-defined and complex problems due to incomplete and imprecise information that characterize the real-world systems. It is therefore suitable for uncertain or approximate reasoning that involves human intuitive thinking. Due to the inherent uncertainty, it seems that Fuzzy sets are more suitable to handle Global risk factors and Acts of God than other approaches. In fact, very often, individuals involved in engineering projects deal with these risks in an approximate manner, using natural language, and not probabilities to assess their likelihood and impacts. Risk management can, to large extent, be considered cognitive in nature. The process of risk management in construction industry is mainly based upon experience, assumptions and human judgment.

Zadeh asserted that fuzzy sets handle vagueness type of uncertainty better than any other approach and vagueness is a distinct type of uncertainty to be treated by probabilistic methods. According to the fuzzy set theory a meaning in natural language is a matter of degree.

Considering one variable of global risk factors a question can be formulated: " is the competition strong?". The answer is not always simply 'yes or no". It largely depends on the subject who is to respond. The meaning of strong is not precise. It varies from individual to individual.

The basic fundamentals of fuzzy set theory are the concepts of linguistic variable and degree of membership. Since most construction engineering decision problems are complex and imprecise, they might be better described by linguistic expressions rather than by numbers. Numbers are associated with precision, whereas decision problems like managing risks need not or do not have specific outcomes but approximate ones. Furthermore, due the imprecision inherent to linguistic expressions the transition from one state to another is smooth. For example, the transition from "very heavy floods" to "heavy floods" is not sharp, but gradual. Linguistic expressions play an important role in this regard because description is at the very core of risk management in construction. Finally, fuzzy sets have the ability to preserve the uncertainty inherent to the problems throughout the analysis instead of making assumptions.

To illustrate the use of fuzzy sets in modeling risk factors a variable designated ' low competition' is chosen as shown is figure 1. The membership functions were derived from interviews with engineering professionals.

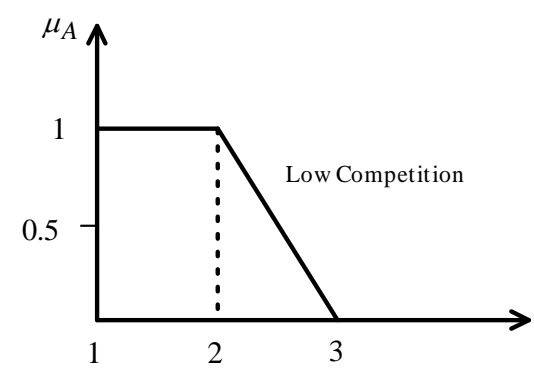

Figure 1: Graphical form of fuzzy set "low competition", where $\mu_{\mathrm{A}}$ is the grade of membership; low competition is fuzzy set $\boldsymbol{A}$; and1, 2 and 3 are the elements of the universe of discourse.

The fuzzy set ''low competition', can also be represented analytically:

$$
A_{\text {low competition }}=[1.0 / 0,0.6 / 2,0.0 / 3]
$$

where 1.0, 0.6 and 0.0 are the degrees of membership. Thus, the membership function of low competition is $[1.0,0.6,0.0]$. 
Apart from low competition other values would be moderate competition and high competition. The corresponding fuzzy sets for these values may be the following:

$B_{\text {moderate competition }}=[0.0 / 2,0.5 / 31.0 / 4,0.5 / 5,0.0 / 6]$;

and

$C_{\text {high competition }}=[0.0 / 5,0.5 / 6,1.0 / 7,1.0 / 8,1.0 / 10]$.

Important to notice, though, are the areas of overlap between low and moderate and moderate and high competition membership functions. These overlaps represent the "grey" zones, that is, the gradual transitions from membership to non-membership properties. In a crisp set an object is either a member of a set or not.

The only grades of membership in the crisp set low competition are 1 and 0 . Thus, competition levels from 0 to 3 are members of the set with a grade 1 and, all other elements of the universe are not, and they have grade membership of 0 . As it can be seen, the change in the grades is quite abrupt.

Using similar reasoning, all global risk factors and Acts of God can be modeled and analyzed based on fuzzy sets. The major challenge lies with the development of membership functions for the different variables. There are various ways for that purpose of which interviews and discussions with project team members is the most prominent. Existing transforming these computations, through reasoning, into judgement, opinions, evaluations and advice, on the other hand, would increase the quality of data processing and thus provide better support to decision-making.

\subsection{Summary}

Fuzzy set theory is not without problems. There is a strong controversy around the fundamentals of probability theory and fuzzy set theory. Some scholars have argued that fuzzy sets theory is no more than a false appearance of probability theory. For most of the critics, fuzzy sets has no well established mathematical or empirical methods to model human judgment as stated by Lootsma ${ }^{15}$. The main sources of the criticisms are probably the fundamentals of fuzzy sets theory which are clearly in contradiction with the dominant scientific view of the world - precision. Detractors of fuzzy set theory argue that it is probability theory in disguise, as stated by Cheseeman ${ }^{16}$.

\section{Summary of the Techniques}

The strengths and weaknesses of the techniques that have been discussed are summarized in Table 1. For each of the four risk analysis techniques, the evaluation of type of risk, prominent uncertainty and tools to be employed are shown.

Table 1: Summary of risk analysis techniques and tools

\begin{tabular}{|c|c|c|c|c|c|c|}
\hline \multirow{3}{*}{ No } & \multirow{3}{*}{ Technique } & \multicolumn{5}{|c|}{ Modeling and Analysis Suitability } \\
\hline & & \multicolumn{3}{|c|}{ Risk Group } & \multirow[t]{2}{*}{ Prominent Uncertainty } & \multirow[t]{2}{*}{ Tools } \\
\hline & & $\begin{array}{c}\text { (A) } \\
\text { Organization } \\
\text {-Specific }\end{array}$ & $\begin{array}{c}\text { (B) } \\
\text { Global }\end{array}$ & $\begin{array}{c}\text { (C) } \\
\text { Acts of } \\
\text { God }\end{array}$ & & \\
\hline 1 & Probability & Very Good & Very Poor & Very Poor & Random $(A)$ & DSS \\
\hline 2 & Certainty Factor & Very Poor & Good & Good & Epistemic ( $B$ and $C$ ) & KBS \\
\hline 3 & $\begin{array}{l}\text { Dempster_Shaffer } \\
\text { Theory of Evidence }\end{array}$ & Poor & Good & Good & Epistemic ( $B$ and $C)$ & KBS \\
\hline 4 & Fuzzy Set Theory & Poor & Very Good & Very Good & Epistemic (B and $C$ ) & KB-DSS \\
\hline
\end{tabular}

procedures and rules for combining membership functions allow performance of complex operations. Furthermore, there are now many software shells that can be used to develop effective models and run analysis. The inputs and outputs of fuzzy set analysis are natural language expressions which facilitate interpretation.

\subsection{Tools}

A combination of knowledge based systems and decision support systems resulting in a KBS-DSS can be a useful strategy when employing fuzzy sets approach. The first part houses the vast quantity of knowledge whereas the second one provides support to decision-makers. The need for a KBDSS stems from the fact that a system capable of processing data and numeric relationships, on the one hand, and
The indications provided in the table constitute a guide on what a specific technique can help to manage. For example, probabilistic modeling can be effectively to model organization specific and global risks factors where the underlying uncertainty is mainly random. For that purpose Decision Support Systems DSS seem to be the most appropriate tools.

On the other hand, probabilistic modeling is very poor in tackling natural events or Acts of God as data for these is scarce and frequently unavailable. The same procedure is followed with regard to other risk analysis techniques.

\section{Conclusions}

The importance of managing construction engineering risk factors has been discussed. An extensive analysis of 
construction projects implementation with emphasis on cost performance has triggered work on risk modeling and analysis. It has been concluded that the nature of risks under consideration is determinant in the selection of modeling and analysis techniques. As it has been shown, not all uncertainty is random in nature. A great deal of engineering management and technical issues do not comply with randomness properties. They are mainly cognitive and thus do not lend themselves to precise measurement.

Three groups of risk factors inherent in construction engineering projects have been presented and explained. It is understood that the nature of the risk factors is diverse and thus their handling requires appropriate techniques and tools. Indeed, the type uncertainty associated with each of the groups differs. While organization-specific risk factors can be effectively modeled through probabilistic analysis, uncertainty underlying global and acts of god risk factors appears to be much more difficult to capture using the same approach. The uncertainty type associated with the latter risk factors derives mainly from reduced knowledge rather than natural variability. As such, epistemic uncertainty handling techniques are likely to be more effective than probabilistic approaches.

Several uncertainty modeling techniques namely uncertainty theory, Dempster_Shaffer Theory of Evidence and Fuzzy Logic have been discussed with emphasis on appropriateness and robustness. Although there is no consensus on the applicability of these techniques to bridge the gap between the ideal and feasible solutions provided by the probabilistic analysis, it appears that they can be employed for such purpose. In particular, Dempster-Shafer Theory and Fuzzy Logic are capable of modeling epistemic uncertainty through belief and membership functions. Some examples of fuzzy logic knowledge-based-systems using inference have been developed. Nevertheless, research is required to ascertain the extent to which these and additional techniques can be applied to improve construction engineering risks modeling and analysis.

\section{References}

1. Thompson, P. and Perry, J., Engineering Construction Risks. A Guide to Project Risk Analysis and Risk Management. (London: Thomas Telford. 1992)

2. Flanagan, R and Norman, G., Risk Management and Construction. (London: Blackwell. 1993)

3. Helton,, J Uncertainty and sensitivity analysis in the presence of stochastic and subjective uncertainty. "Journal of Statistical Computation and Simulation” 57: 3-76 (1997).

4. Klir, G. Where Do We Stand on Measures of Uncertainty, Ambiguity, Fuzziness and the Like?. Fuzzy Sets and Systems, 1987, 24, pp.141-160.

5. Van Gelder, P.H.A.J.M., "Statistical Methods for the RiskBased Design of Civil Structures", 2000, Communications on Hydraulic and Geotechnical Engineering, ISSN:0169-6548001, 248pages.

6. Zadeh, L. A., Fuzzy Sets. Information and Control, 1965, 8, pp.338-353

7. Zimmermann, H. J. and Haley, K.B. Theory and Applications of Fuzzy Sets. Operational Research, 78, (Amsterdam: NorthHolland. 1996)

8. Uher, T., Probabilistic Estimating. Cost Engineering, 1996, 38(4), pp. 33-40.

9. Ian Bremmer, "How to Calculate Political Risk," Inc. Magazine, April 2007, p. 101

10. Duda, R. O. et al Development of PROSPECTOR System for Mineral Exploration. Final Report, SRI Projects 5821 and 6415, (Menlo Park, CA, 1979).

11. Clark, D.A., Numerical and Symbolic Approaches to Uncertainty Management. In AI: Artificial Intelligence,, 1990 4, pp.59-91.

12. Blockley, D. I. And Baldwin, J.F., Uncertain Inference in Knowledge-Based Systems. ASCE, Journal of Engineering Mechanics, 1987, VII(3),

13. Shafer, G., Mathematical Theory of Evidence. (Princeton: Princeton University Press,,1976).

14. Zadeh, L. A. (1978) A Meaning Representation Language for Natural Languages. International J. of Man-Machine Studies, 10(4), pp. 395-460.

15. Lootsma, F.A. (1997) Fuzzy Logic for Planning and Decision Making. The Netherlands: Kluwer.

16. Cheeseman, P. C. (1965) In Defence of Probability. Proceedings, International Joint Conference on AI,, pp.10021009. 